\title{
Phase variation of lic1A, lic2A and lic3A in colonization of the nasopharynx, bloodstream and cerebrospinal fluid by Haemophilus influenzae type b
}

\author{
Sarah L. Hosking, † Jane E. Craig and Nicola J. High
} Author for correspondence: Nicola J. High. Tel: +44 161275 5749. Fax: +44 1612755656.
e-mail: nicky.high@man.ac.uk

University of Manchester, School of Biological Science, 1.800 , Stopford Building, Manchester M13 9PT, UK

\begin{abstract}
The role of phase variation of lic1A, lic2A and lic3A in the ability of Haemophilus influenzae type $b$ to colonize the nasopharynx, bloodstream and cerebrospinal fluid (CSF) of infants was investigated. This was achieved by using PCR to determine the number of 5'-CAAT-3' repeats present in each gene, which is indicative of whether each ORF can be expressed. Multiple PCR products of different intensities were amplified from all three genes at each site sampled. This indicated that the nasopharynx, bloodstream and CSF were colonized by a heterogeneous population of organisms, expressing different combinations of lic genes. At each site however, a predominant PCR product was amplified from each gene, indicating that organisms with this genotype were the most abundant. The number of $5^{\prime}$-CAAT-3' repeats in this predominant product varied depending upon whether organisms were isolated from the nasopharynx, bloodstream or CSF. These observations suggest that the expression of different combinations of lic genes may influence the efficiency with which $H$. influenzae colonizes the nasopharynx, bloodstream and CSF of infant rats.
\end{abstract}

Keywords: Haemophilus influenzae type b, phase variation, invasive disease, lic genes.

\section{INTRODUCTION}

Haemophilus influenzae type $\mathrm{b}$ is an encapsulated organism which causes invasive disease, such as meningitis (reviewed by Moxon, 1985). It is also a frequent cause of both upper and lower respiratory tract infections. LPS is major virulence determinant of $H$. influenzae and was the first cell surface structure expressed by this organism that was shown to exhibit phase variation. LPS phase variation in $H$. influenzae is characterized by the spontaneous loss and gain of oligosaccharide structures present in the outer core (Kimura \& Hansen 1986). Mechanisms contributing to LPS phase variation were first identified in the gene lic1 A which contains multiple copies of the tandem repeat $5^{\prime}$ CAAT- $3^{\prime}$ within the $5^{\prime}$ end of its coding sequences †Present address: Unilever Research, Port Sunlight, Quarry Road East,
Wirral L63 3JW, UK.

Abbreviations: CSF, cerebrospinal fluid; i.n., intranasal; i.p., intraperitoneal.
(Weiser et al., 1989). lic1A was subsequently shown to encode phosphorylcholine kinase (ChoP) which promotes the phase-variable substitution of LPS with choline (Weiser et al., 1997). Intragenic 5'-CAAT-3' repeats were also identified in two further genes, lic $2 A$ and lic3A (Weiser et al., 1990). lic2A encodes a glycosyl transferase which is required for the phase-variable expression of the Gal $\alpha(1-4) \beta$ Gal LPS structure (High et al., 1993). However, it is the product of $\lg t C$, another phase-variable gene, that ultimately dictates whether this structure is synthesized (Hood et al., 1996). The function of the product of $\operatorname{lic} 3 \mathrm{~A}$ has not been determined and there is no evidence, to date, to suggest that it plays a role in LPS biosynthesis (Maskell et al., 1992). The phase-variable expression of these genes is generated by slipped-strand mispairing (Levinson \& Gutman, 1987), which causes the number of $5^{\prime}$-CAAT- $3^{\prime}$ repeats to vary from generation to generation. This results in translational frameshifting, placing upstream initiation codons in- and out-of-frame with the ORF. Through this process the expression of these genes can be reversibly switched on and off (Weiser et al., 1989). 
Analysis of the genome sequence of $H$. influenzae strain $\mathrm{Rd}$ has identified a further nine loci which contain multiple repeats of a tetrameric sequence other than $5^{\prime}$ CAAT-3' (Hood et al., 1996). Tandem repeats of 5'GCAA- $3^{\prime}$ have also been found in genes involved LPS biosynthesis (Jarosik \& Hansen, 1994). All of these genes encode candidate virulence genes, indicating that $H$. influenzae has a massive potential for variation during the course of infection which may contribute to its success as a pathogen. The significance of the phase variation of each of these genes in the pathogenesis of invasive disease is unknown. In the case of LPS, phase variation is thought to enable $H$. influenzae to evade antigen-specific host immune defences by promoting variation in cell surface composition. The ability to vary the expression of multiple virulence determinants may optimize the virulence potential of $H$. influenzae by enabling the expression of the most appropriate phenotypic attributes for a given environment, or stage in pathogenesis. This may be of particular significance in the development of invasive disease which, in the case of meningitis, requires translocation of $H$. influenzae from the nasopharynx to the central nervous system. This sequence of events involves the colonization of several distinct environments and the interaction of the organism with a variety of different host cells.

In this paper we investigate the role of the phase variation of $l i c 1 A, l i c 2 A$ and $l i c 3 A$ in colonization of the major compartments colonized by $H$. influenzae during the development of invasive disease. This was achieved by monitoring the number of $5^{\prime}$-CAAT-3' repeats present in each gene from organisms isolated from the nasopharynx, bloodstream and cerebrospinal fluid (CSF) of infant rats infected with $H$. influenzae strain Eagan. Organisms colonizing each of these environments were shown to express different combinations of lic genes. Phase variation of these genes may therefore play an important role in facilitating the survival and persistence of $H$. influenzae in the diverse environments encountered during the development of invasive disease.

\section{METHODS}

Bacterial strains and culture conditions. H. influenzae strain Eagan was used throughout this study (Anderson et al., 1980). H. influenzae was grown in brain-heart infusion broth (BHI) supplemented with haemin $\left(10 \mu \mathrm{g} \mathrm{ml}^{-1}\right)$ and NAD $\left(2 \mu \mathrm{g} \mathrm{ml}^{-1}\right)$. Solid BHI medium was prepared by the addition of Bacto Agar $(1 \%, \mathrm{w} / \mathrm{v})$ and Levinthals base $(10 \%, \mathrm{v} / \mathrm{v})$ (Alexander, 1965).

The infant rat model of $\boldsymbol{H}$. influenzae invasive disease. Natural litters of 5-d-old Sprague-Dawley rats were reduced to 12 rats at birth and randomized. Infant rats were challenged with $H$. influenzae strain Eagan by intranasal (i.n.) inoculation so that the expression status of lic genes during colonization of the nasopharynx could be established (Moxon et al., 1974). Although invasive disease occurs following i.n. challenge the level of bacteraemia generated is often low and does not always lead to meningitis. A separate group of animals were therefore challenged intraperitoneally (i.p.) to enable efficient isolation of organisms from the bloodstream and CSF (Smith et al., 1973). For both i.n. and i.p. challenges the inocula were prepared and quantified as described by Moxon et al. (1974) and Smith et al. (1973), and adjusted to the desired concentration. After $48 \mathrm{~h}$ approximately $20 \mu \mathrm{l}$ blood was taken from a tail vein of which $5 \mu \mathrm{l}$ was plated onto supplemented BHI agar to detect and quantify bacteraemia. CSF was also removed from each animal by cisterna magna puncture and $10 \%$ of the volume obtained plated onto supplemented BHI agar. Bacteria colonizing the nasopharynx were harvested by washing one nostril with $50 \mu \mathrm{l}$ PBS and then withdrawing approximately $20 \mu \mathrm{l}$ from the opposite nostril. Two microlitres of each sample was cultured as described above, in the presence of bacitracin $\left(1 \mathrm{mg} \mathrm{ml}^{-1}\right)$.

PCR amplification of the $5^{\prime}$-CAAT-3' regions of lic1A, lic2A and lic3A. Blood, CSF and nasal washings were boiled for 5 min. Whole-cell debris was removed by centrifugation and the supernatant decanted to a fresh tube. Samples were then treated with Hybaid pre-amplification reagent, according to the manufacturer's recommendations, to remove compounds which might inhibit the PCR. The PCR was carried out in a buffer containing $500 \mathrm{mM} \mathrm{KCl}, 100 \mathrm{mM}$ Tris/ $\mathrm{HCl}, \mathrm{pH} 8$, $0 \cdot 1 \%(\mathrm{w} / \mathrm{v})$ gelatin and $25 \mathrm{mM} \mathrm{MgCl}{ }_{2} \cdot\left[\alpha^{-}{ }^{32} \mathrm{P}\right] \mathrm{dCTP}[10 \mu \mathrm{Ci}$ $(0.37 \mathrm{MBq})]$ was added to the dNTP mixture to increase the sensitivity of the PCR. The $5^{\prime}$-CAAT-3' region from lic1A was amplified using 5'-GGAATGGAATGCTGATGAAG-3' and 5'-TCATAAGATTCAGAGCCT-3' or, in the case of nasal lavage samples, 5'-TCTTTCAGCTAACCGAGC-3'. The 5'CAAT-3' region from lic $2 A$ was amplified using $5^{\prime}$-GCAACTGAACGTCGCAAACAT- $3^{\prime}$ and 5'-CACACACTTATTCCATAATAAG- $3^{\prime}$. The primers used to amplify the $5^{\prime}$-CAAT3' from lic3A were 5'-CGGAGATAGTACAACTGATA-3' and $5^{\prime}$-AACTTGTTCCATTACCTGCA-3'. The reaction mixture was incubated at $94^{\circ} \mathrm{C}$ for 5 min and then 45 cycles of PCR were carried out, each cycle consisting of $94^{\circ} \mathrm{C}$ for $1 \mathrm{~min}$, $57^{\circ} \mathrm{C}$ for $1 \mathrm{~min}$ and $72{ }^{\circ} \mathrm{C}$ for $1 \mathrm{~min}$. The resultant PCR products were analysed by electrophoresis through a $6 \%$ polyacrylamide gel. The size of each product and hence the number of $5^{\prime}$-CAAT-3' repeats was determined by comparison with an M13 sequencing ladder. To confirm the predicted number of $5^{\prime}$-CAAT $-3^{\prime}$ repeats a number of samples from each PCR set was sequenced directly as previously described (High et al., 1993). Autoradiographs were scanned using a Phoretix densitometer and the captured images analysed using Phoretix ID gel analysis software. Bands were identified and densitometry measurements obtained after an automatic background subtraction had been performed.

Statistical analysis. Densitometry measurements obtained for each set of PCR products were compared statistically. PCR products derived from lic1A, which comprised two bands, were compared using a Mann-Whitney non-parametric test. PCR products derived from lic2 $A$ and lic $3 A$, which comprised three bands, were compared using the Kruskal-Wallis nonparametric test. In both cases $P$ values $<0.05$ were considered significant.

\section{RESULTS}

\section{Phase variation of lic1A in $\mathrm{H}$. influenzae colonizing} the nasopharynx, bloodstream and CSF of infant rats

The distribution of $5^{\prime}$-CAAT-3' repeats in lic1 A, lic2A and lic $3 A$ present in organisms isolated from the nasopharynx, bloodstream and CSF of infected animals was determined. Multiple PCR products of different intensities were obtained and their relative abundance determined by densitometry (Figs $1 \mathrm{~b}, 2 \mathrm{~b}$ and $3 \mathrm{~b}$ ). In the case of lic1A, which encodes ChoP, two PCR products 
(a)

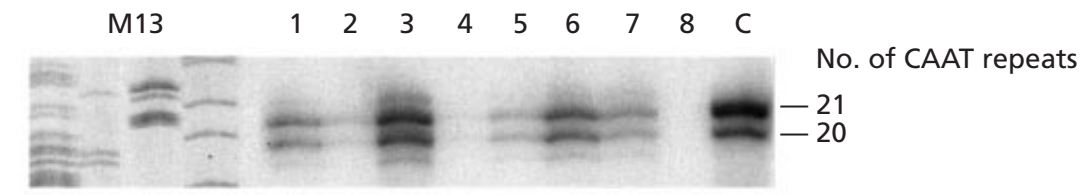

(b)

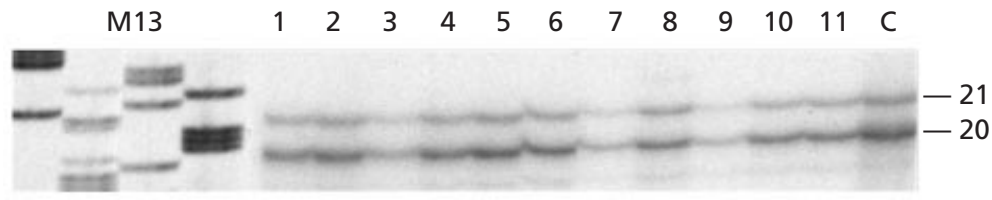

(c)
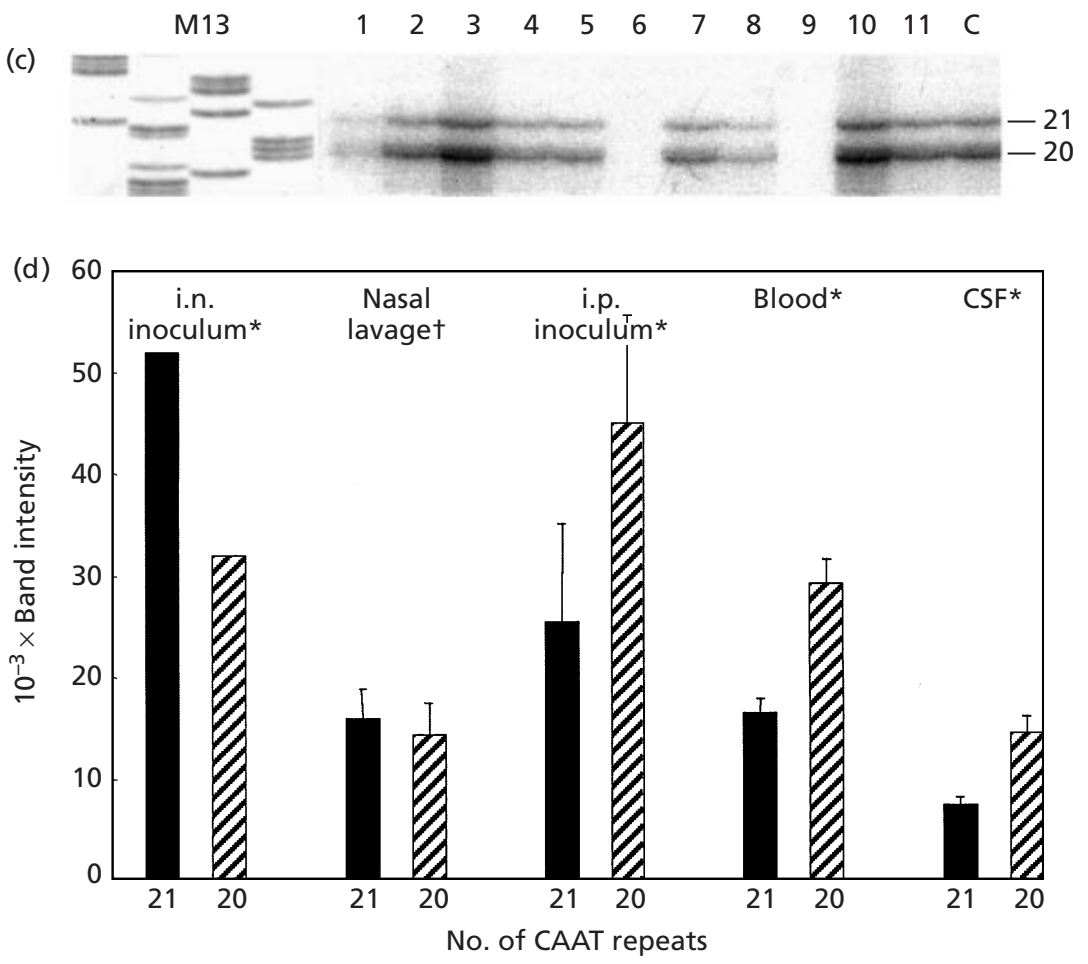

Fig. 1. The distribution of lic1A genotypes in $H$. influenzae strain Eagan isolated from the nasopharynx, bloodstream and CSF of infant rats. The $5^{\prime}$-CAAT- $3^{\prime}$ repeat region in lic1A was amplified by PCR and the distribution of PCR products visualized by PAGE. In all experiments each lane represents PCR products obtained from an individual animal. Lane $C$ represents PCR products amplified from broth-grown organisms used as the inoculum. (a) PCR products amplified from nasal lavage samples obtained from animals challenged by i.n. inoculation. (b, c) PCR products amplified from blood and CSF samples, respectively, obtained from animals challenged by i.p. inoculation. Lanes with corresponding numbers represent paired blood/CSF samples obtained from the same animal. (d) The intensities of each of the PCR products shown in (a)-(c) were determined by densitometry using the Phoretix software package. The mean intensity for bands containing the same number of 5'-CAAT-3' repeats was calculated for each sample. These values are presented as a histogram. A Mann-Whitney non-parametric test was used to determine whether the relative quantities of PCR products in each sample were significantly different $(P<0.05)$ * indicates samples where $P<0.05$; + indicates samples where $P>0.05$ and no significant difference was detected.

of comparable intensity $(P>0 \cdot 05)$ were amplified from nasal lavage samples $48 \mathrm{~h}$ post-infection (Fig. 1a, lanes 1-8; Fig. 1b). One PCR product contained 20 copies of 5'-CAAT-3', which is non-permissive for expression of lic1A. The second PCR product contained 21 copies of 5'-CAAT-3', which is consistent with expression of the ORF and phosphorylcholine substitution of LPS (Weiser et al., 1989, 1997). In the original inoculum, the PCR product containing 21 copies of $5^{\prime}$-CAAT- $3^{\prime}$ was most abundant, indicating that during nasopharyngeal colonization there had been a shift in the population such that this genotype was no longer predominant (Fig. 1, lane C). In blood and CSF samples, from animals challenged via the i.p. route, the distribution of PCR products amplified was different from that obtained from nasopharyngeal lavages (Fig. 1a and b). In these samples the PCR product containing 20 copies of $5^{\prime}$ CAAT $-3^{\prime}$ was consistently more abundant. An identical distribution of PCR products was also obtained from the broth-grown organisms used as the inoculum.

\section{Phase variation of lic2A in $\mathrm{H}$. influenzae colonizing the nasopharynx, bloodstream and CSF of infant rats}

In all three sites sampled the predominant number of $5^{\prime}$ CAAT-3' repeats in lic $2 A$ was consistent with the expression of a full-length ORF (High et al., 1993). The pattern of PCR products amplified from nasal lavage samples was identical to that obtained from the original inoculum. The predominant PCR product amplified contained 17 copies of $5^{\prime}$-CAAT-3' (Fig. 2a, lanes 1-8; Fig. 2b). This number of repeats in lic $2 A$ is associated with high-level reactivity to $\mathrm{mAb} 4 \mathrm{C} 4$ which recognizes the phase variable Gal $\alpha(1-4) \beta$ Gal LPS structure (High et al., 1993). A second PCR product containing 18 copies of $5^{\prime}$-CAAT-3', which is not consistent with expression of $\operatorname{lic} 2 \mathrm{~A}$, was also amplified, but was much less abundant. In some samples a third PCR product, corresponding to 16 copies of $5^{\prime}$-CAAT-3', was also detected. This format is also consistent with expression of $l i c 2 A$ but is associated with a lower level of reactivity 
(a)

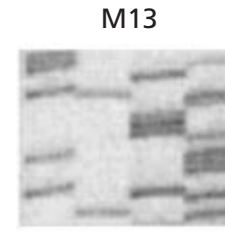

$\begin{array}{lllllllll}C & 1 & 2 & 3 & 4 & 5 & 6 & 7 & 8\end{array}$

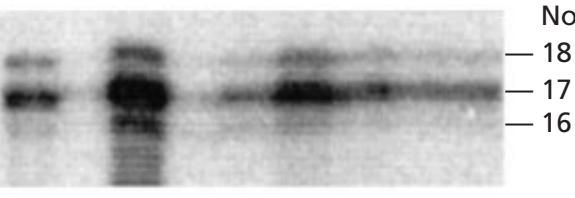

(b)

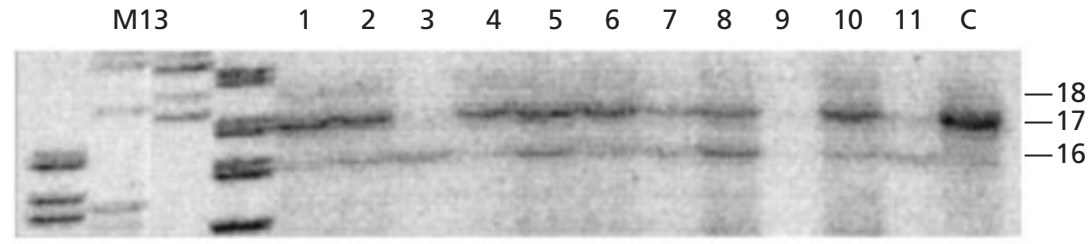

(c)
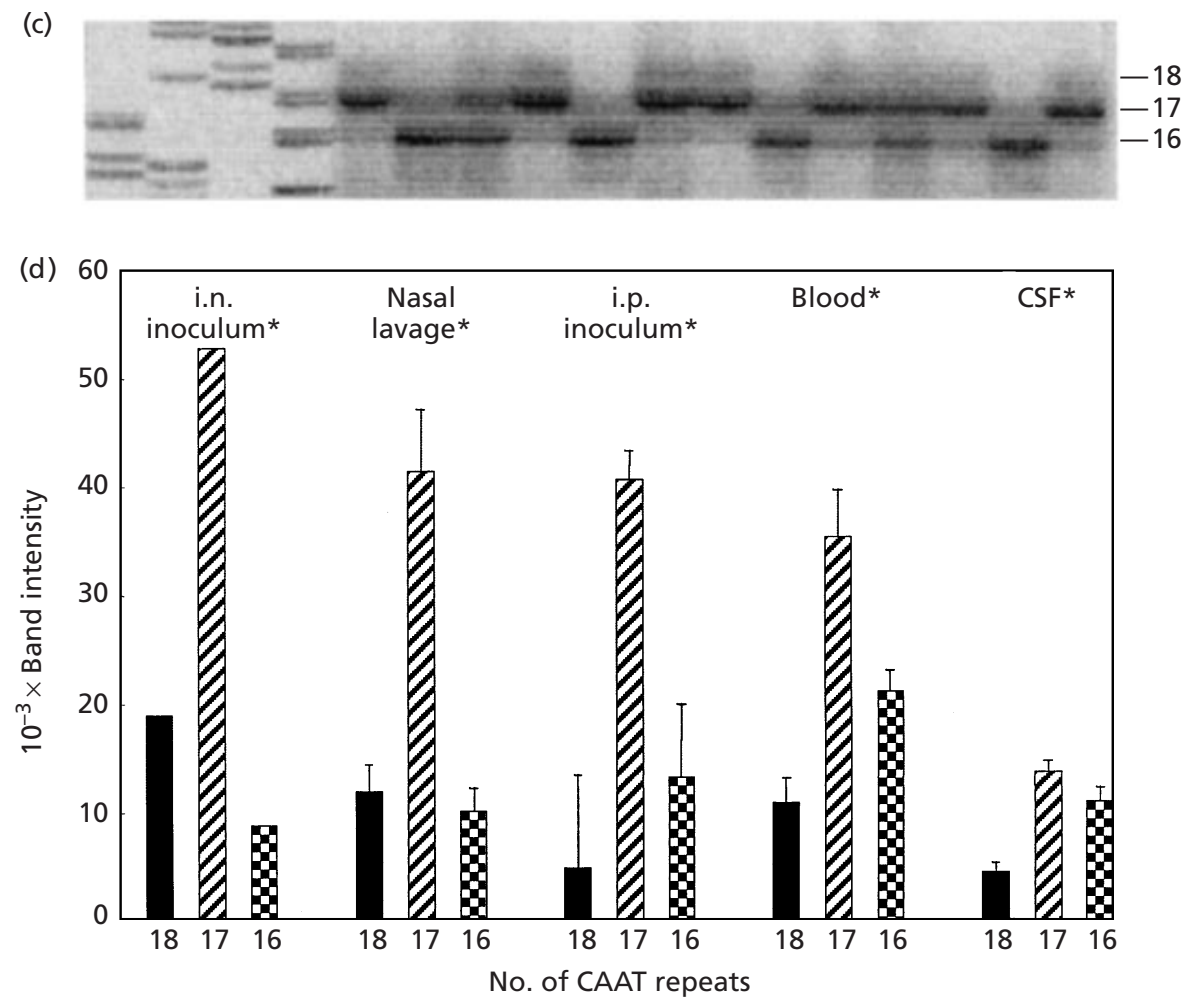

Fig. 2. The distribution of lic $2 A$ genotypes in $\mathrm{H}$. influenzae strain Eagan isolated from the nasopharynx, bloodstream and CSF of infant rats. The 5'-CAAT-3' repeat region in lic $2 A$ was amplified by PCR and the distribution of PCR products visualized by PAGE. In all experiments each lane represents PCR products obtained from an individual animal. Lane $C$ represents PCR products amplified from brothgrown organisms used as the inoculum. (a) PCR products amplified from nasal lavage samples obtained from animals challenged by i.n. inoculation. (b, $c$ ) PCR products amplified from blood and CSF samples, respectively, obtained from animals challenged by i.p. inoculation. Lanes with corresponding numbers represent paired blood/CSF samples obtained from the same animal. (d) The intensities of each of the PCR products shown in (a)-(c) were determined by densitometry using the Phoretix software package. The mean intensity for bands containing the same number of 5'-CAAT-3' repeats was calculated for each sample. These values are presented as a histogram. A Kruskal-Wallis non-parametric test was used to determine whether the relative quantities of PCR products in each sample were significantly different $(P<$ 0.05). * indicates samples where $P<$ 0.05 . to mAb 4C4. PCR amplification of blood and CSF samples, from i.p.-challenged animals, revealed that in these environments the majority of organisms had either 17 or 16 copies of $5^{\prime}$-CAAT- $3^{\prime}$ and therefore had the potential to express $\operatorname{lic} 2 A$ and incorporate $G a l \alpha(1-4)$ $\beta$ Gal into their LPS (Fig. 2) (High et al., 1993). PCR products containing a non-permissive number of $5^{\prime}$ CAAT $-3^{\prime}$ repeats were also detected, but were approximately three times less abundant than products amplified from organisms containing 17 copies of $5^{\prime}$ CAAT-3'. The apparent ratio of the PCR products obtained from blood samples was, in some cases, different from the original inoculum, in which the predominant PCR product amplified contained 17 copies of $5^{\prime}$-CAAT-3'. This was particularly apparent in samples 3, 5, 8 and 11 (Fig. 2) in which PCR products containing 16 copies of $5^{\prime}$-CAAT-3' were amplified, a format which was not readily detectable in the original inoculum. This suggested that phase variation had occurred in vivo, following i.p. challenge, but that the ability to express lic $2 \mathrm{~A}$ had been retained. Comparison of the PCR products obtained from paired blood and CSF samples revealed that translocation of organisms from the bloodstream to the central nervous system had occurred concurrently with a change in the predominant number of $5^{\prime}$-CAAT-3' repeats in some animals. In animals 2, 5 and 8 the predominant number of repeats changed from 16 to 17 . In animals 7, 9 and 11 the switch was in the opposite direction (Fig. 2). In each case the observed change did not alter the ability to express lic2A. PCR products generated from organisms unable to express lic $2 A$ were consistently less abundant in each CSF sample. The fact that phase variation had occurred during translocation of organisms from the bloodstream 
(a)

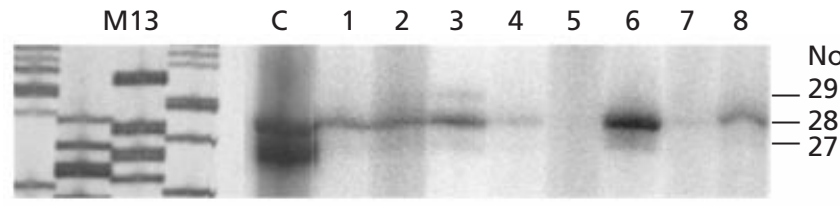

(b)
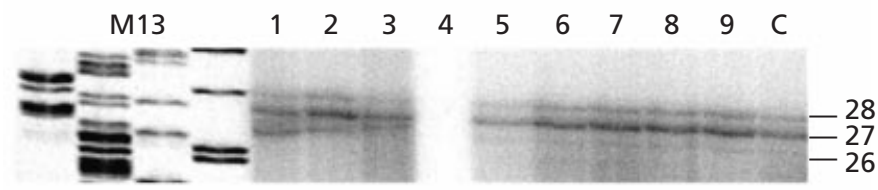

(c)

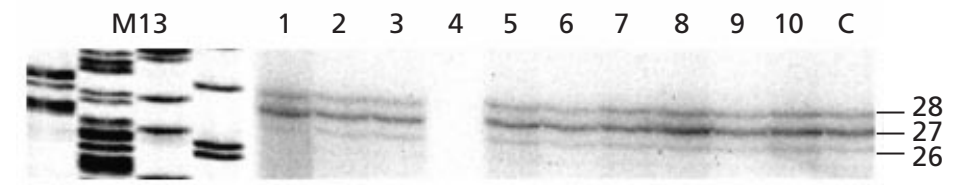

(d)

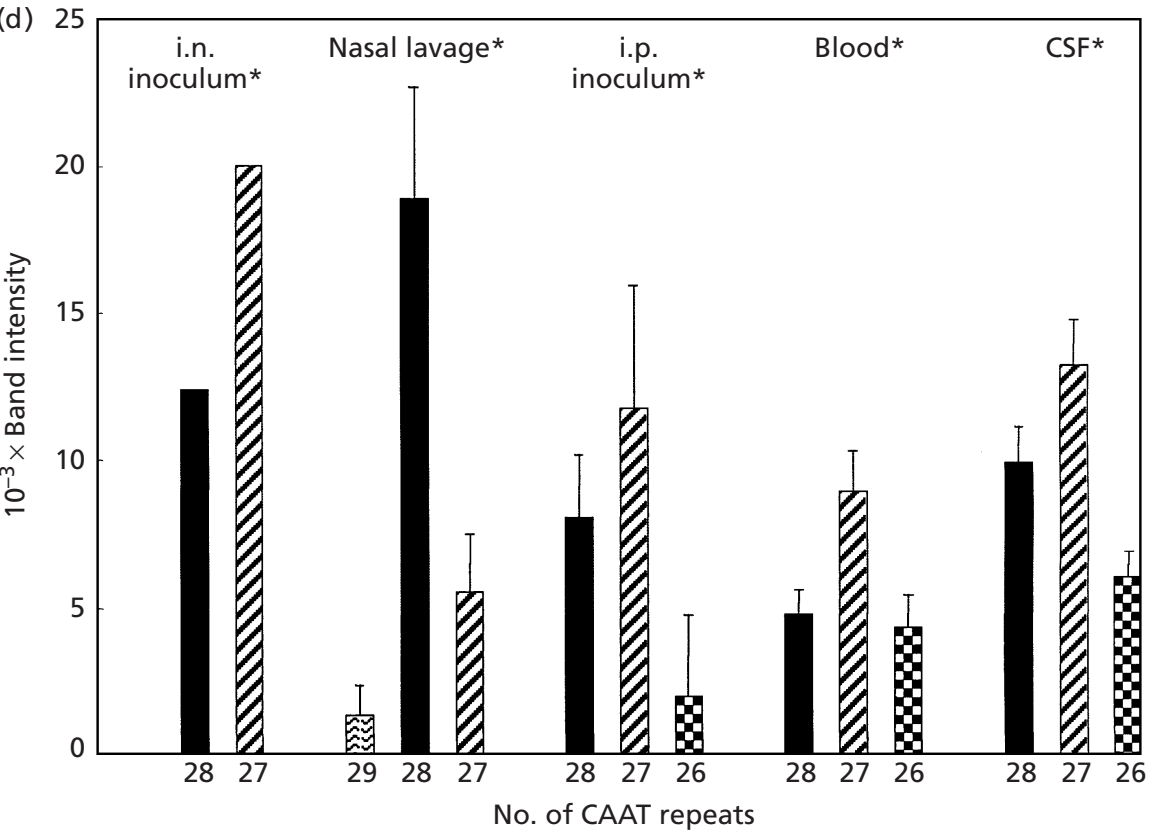

Fig. 3. The distribution of lic3A genotypes in $H$. influenzae strain Eagan isolated from the nasopharynx, bloodstream and CSF of infant rats. The 5'-CAAT-3' repeat region in lic3A was amplified by PCR and the distribution of PCR products visualized by PAGE. In all experiments each lane represents PCR products obtained from an individual animal. Lane $C$ represents PCR products amplified from broth-grown organisms used as the inoculum. (a) PCR products amplified from nasal lavage samples obtained from animals challenged by i.n. inoculation. (b, c) PCR products amplified from blood and CSF samples, respectively, obtained from animals challenged by i.p. inoculation. Lanes with corresponding numbers represent paired blood/CSF samples obtained from the same animal. (d) The intensities of each of the PCR products shown in (a)-(c) were determined by densitometry using the Phoretix software package. The mean intensity for bands containing the same number of $5^{\prime}$-CAAT-3' repeats was calculated for each sample. These values are presented as a histogram. A Mann-Whitney non-parametric test was used to determine whether the relative quantities of PCR products in each sample were significantly different $(P<$ 0.05). * indicates samples where $P<0.05$. to the CSF suggests that organisms with this genotype were selected against.

\section{Phase variation of lic3A in $\boldsymbol{H}$. influenzae colonizing the nasopharynx, bloodstream and CSF of infant rats}

Amplification of the 5'-CAAT-3' repeats in lic $3 A$ from organisms present in nasal lavage samples generated a predominant PCR product consistent with the presence of 28 repeat units in this gene (Fig. 3a, lanes 1-8; Fig. $3 \mathrm{~b})$. This number of $5^{\prime}$-CAAT-3' repeats is permissive for the expression of lic3A (Maskell et al., 1992). PCR products corresponding to 29 and 27 repeat units were also detectable in one sample (Fig. 3, lane 3), but were present at low levels. In the inoculum used to challenge these animals the majority of organisms contained 27 copies of $5^{\prime}$-CAAT-3', which is non-permissive for expression of $\operatorname{lic} 3 A$, although a subpopulation containing 28 copies was also present. Colonization of the nasopharynx by $H$. influenzae therefore appears to involve selection of organisms which have the ability to express lic $3 \mathrm{~A}$. In contrast, in the bloodstream and CSF the predominant population of organisms were unable to express lic3A. Three PCR products were amplified from blood and CSF samples (Fig. $3 a$ and b). The relative distribution of these products was identical to that of organisms in the original inoculum. The most intense PCR product was consistent with the presence of 27 copies of $5^{\prime}$-CAAT- $3^{\prime}$ in lic3A, which does not permit translation of the ORF. Subpopulations of organisms, containing 28 and 26 copies of $5^{\prime}$-CAAT-3', both of which permit expression of $\operatorname{lic} 3 A$, were also detected at low levels.

\section{DISCUSSION}

In this study we have investigated the role of the phasevariable expression of $\operatorname{lic} 1 \mathrm{~A}$, lic2 $\mathrm{A}$ and $\operatorname{lic} 3 \mathrm{~A}$ in coloni- 
zation of the nasopharynx, bloodstream and CSF of infant rats infected with $H$. influenzae type b strain Eagan. Each site was found to be colonized by a heterogeneous population of organisms, expressing different combinations of each gene product. This is consistent with the idea that phase variation is a stochastic process. Homogeneous populations would only be anticipated to occur in the presence of a strong selection pressure which favoured the expression of a particular combination of lic genes. The relative proportions of each lic genotype differed most between organisms colonizing the nasopharynx and organisms isolated from either the bloodstream or CSF. In the latter two environments the pattern of PCR products generated was indistinguishable. This may reflect the similar distribution of complement, antibodies and other components of the immune system in these two locations once an inflammatory response has occurred (Zwahlen et al. 1982; Whittle \& Greenwood, 1977). The observed differences between the lic genotypes harboured in these environments suggests that $H$. influenzae may require different phenotypic attributes for colonization of the nasopharynx, bloodstream and CSF.

Evidence of a selection pressure influencing the distribution of lic genotypes during nasopharyngeal colonization was most apparent in the case of lic3A. During the $48 \mathrm{~h}$ period following i.n. challenge the predominant lic3A genotype changed, relative to the inoculum, resulting in a marked reduction in the proportion of organisms unable to express lic $3 A$. This observation suggests that expression of this gene is important for nasopharyngeal colonization, although its precise role has yet to be determined. The distribution of lic $1 \mathrm{~A}$ genotypes also altered following i.n. challenge. However, this did not result in colonization by a predominant population since equal proportions of organisms which either expressed or did not express lic1A were detected. This even distribution of genotypes would suggest that during the first $48 \mathrm{~h}$ the expression status of $l i c 1 \mathrm{~A}$ is not important for nasopharyngeal colonization. In a study by Weiser et al. (1998), a gradual increase in the proportion of organisms able to express $\operatorname{lic} 1 \mathrm{~A}$ was shown to occur in the nasopharynx over a period of $10 \mathrm{~d}$. If the distribution of lic1A genotypes had been monitored over a longer period, a gradual increase in the proportion of organisms expressing this gene might therefore have been expected to occur. In contrast to lic1 $A$ and $\operatorname{lic} 3 A$, the distribution of lic $2 A$ genotypes in the nasopharynx was indistinguishable from that of the original inoculum. No conclusive evidence suggesting that organisms expressing this gene were selected in the nasopharynx was therefore obtained.

Following i.p. challenge, differences were observed in the distribution of $5^{\prime}$-CAAT- $3^{\prime}$ repeats in blood and CSF samples, relative to that in the original inoculum. The numbers of $5^{\prime}$-CAAT-3' repeats also varied between paired blood and CSF samples, indicating that phase variation of lic2A had occurred in vivo. Despite phase variation organisms expressing lic $2 A$ always formed the predominant population suggesting that this phenotype was important in the persistence of $H$. influenzae in the bloodstream and CSF. This notion is consistent with previous observations that show that $H$. influenzae mutants which lack a functional copy of lic $2 \mathrm{~A}$ have reduced virulence in the infant rat model (Cope et al., 1991). No evidence was obtained, however, that phase variation followed by selection of specific lic1 $A$ and lic3A genotypes had occurred. The distribution of genotypes in organisms present in the bloodstream and CSF was indistinguishable from that of broth grown organisms used as the inoculum. If a specific genotype was essential for survival at these sites, the relative proportion of organisms with this attribute would be expected to increase. This was not the case for either lic1 A or lic 3A. Expression of these genes may therefore be neither an advantage nor a disadvantage in these environments.

In summary, the phase variation of lic genes has been monitored in vivo using an infant rat model of $H$. influenzae invasive disease. We have demonstrated that organisms colonizing the nasopharynx express a different combination of lic genes to those colonizing the bloodstream and CSF. Phase variation of these genes may therefore play an important role in the development of invasive disease by $H$. influenzae.

\section{ACKNOWLEDGEMENTS}

This work was funded by a project grant from the Wellcome Trust.

\section{REFERENCES}

Alexander, H. E. (1965). The Haemophilus group. In Bacterial and Mycotic Infections of Man, pp. 724-741. Edited by R. H. Dubos \& J. G. Hirsch. London: Pitman Medical Publishing.

Anderson, P., Flesher, A., Shaw, S., Harding, L. \& Smith, D. H. (1980). Phenotypic and genetic variation in the susceptibility to Haemophilus influenzae type b to antibodies to somatic antigens. J Clin Invest 65, 885-891.

Cope, L. D., Jogev, R., Mertsola, J., Latimer, J. L., Hanson, M. S., McCracken, G. H. \& Hansen, E. J. (1991). Molecular cloning of a gene involved in lipooligosaccharide biosynthesis and virulence expression by Haemophilus influenzae type b. Mol Microbiol 5, 1113-1124.

High, N. J., Deadman, M. E. \& Moxon, E. R. (1993). The role of a repetitive DNA motif $\left(5^{\prime}\right.$-CAAT- $\left.3^{\prime}\right)$ in the variable expression of the Haemophilus influenzae lipopolysaccharide epitope Gal$\alpha(1-4) \beta$ Gal. Mol Microbiol 9, 1275-1282.

Hood, D. W., Deadman, M. E., Jennings, M. P., Bisercic, M., Fleischmann, R. D., Venter, J. C. \& Moxon, E. R. (1996). DNA repeats identify novel virulence genes in Haemophilus influenzae. Proc Natl Acad Sci USA 93, 11121-11125.

Jarosik, G. P. \& Hansen, E. J. (1994). Identification of a new locus involved in the expression of Haemophilus influenzae type $\mathrm{b}$ lipooligosaccharide. Infect Immun 62, 4861-4867.

Kimura, A. \& Hansen, E. J. (1986). Antigenic and phenotypic variants of Haemophilus influenzae type b lipopolysaccharide and their relationship to virulence. Infect Immun 51, 60-79.

Levinson, G. \& Gutman, G. A. (1987). High frequencies of short frameshifts in poly-CA/TG tandem repeats borne by bacterio- 
phage M13 in Escherichia coli K-12. Nucleic Acids Res 15, 5323-5338.

Maskell, D. J., Szabo, M. J., Butler, P. D., Williams, A. E. \& Moxon, E. R. (1992). Molecular analysis of a complex locus from Haemophilus influenzae involved in phase-variable lipopolysaccharide biosynthesis. Mol Microbiol 5, 1013-1022.

Moxon, E. R. (1985). Haemophilus influenzae. In Principles and Practice of Infectious Disease, pp. 1274-1279. Edited by G. Mandrell, R. Douglas \& J. Bennet. New York: Wiley.

Moxon, E. R., Smith, A. L., Averill, D. R. \& Smith, D. H. (1974). Haemophilus influenzae meningitis in infant rats after intranasal inoculation. J Infect Dis 129, 154-162.

Smith, A. L., Smith, D. H., Averill, D. R. \& Moxon, E. R. (1973). Production of Haemophilus influenzae type B meningitis in infant rats by intraperitoneal inoculation. Infect Immun 8, 278-290.

Weiser, J. N., Love, J. \& Moxon, E. R. (1989). The molecular mechanism of phase-variation in Haemophilus influenzae lipopolysaccharide. Cell 59, 657-665.

Weiser, J. N., Maskell, D. J., Butler, P. D. \& Moxon, E. R. (1990). Characterisation of repetitive sequences controlling phase vari- ation of Haemophilus influenzae lipopolysaccharide. J Bacteriol 172, 3304-3309.

Weiser, J. N., Shchepetov, M. \& Chong, S. T. (1997). Decoration of lipopolysaccharide with phosphorylcholine: a phase-variable characteristic of Haemophilus influenzae. Infect Immun 65, 943-950.

Weiser, J. N., Pan, N., McGowan, K. L., Musher, D., Martin, A. \& Richards, J. (1998). Phosphorylcholine on the lipopolysaccharide of Haemophilus influenzae contributes to persistence in the respiratory tract and sensitivity to serum killing mediated by Creactive protein. J Exp Med 187, 631-640.

Whittle, H. C. \& Greenwood, B. M. (1977). Cerebrospinal fluid immunoglobulins and complement in meningococcal meningitis. J Clin Pathol 31, 213-216.

Zwahlen, A., Nydegger, U. E., Vaudaux, P., Lambert, P.-H. \& Waldvogel, F. A. (1982). Complement-mediated opsonic activity in normal and infected human cerebrospinal fluid: early response during bacterial meningitis. J Infect Dis 145, 635-646.

Received 14 December 1998; revised 30 June 1999; accepted 8 July 1999. 